\title{
La relación género y cooperativa en Cuba. Perspectivas dentro del marco jurídico experimental de la cooperativa
}

\author{
Orisel Hernández Aguilar* \\ Universidad de Pinar del Río (Cuba)
}

Sumario: I. Una introducción necesaria. II. Potencialidades de las cooperativas para desarrollar un adecuado enfoque de género. III. Situación actual de la relación género y cooperativa en Cuba. IV. Marco jurídico experimental de la cooperativa en Cuba. Posible incidencia en la relación género y cooperativa. V. A modo de conclusión. V. Bibliografía.

Resumen: El objetivo de este trabajo es aportar algunas valoraciones en torno a la actual situación de la relación género y cooperativa dentro de los marcos normativos en los que se desarrolla la cooperativa en Cuba, así como sus potencialidades ante una regulación legal que instrumente una ampliación de dichas prácticas, como la que actualmente se ha promulgado con carácter experimental, como parte de la reestructuración del modelo económico previsto en los Lineamientos de la Política Económica y Social del Partido y la Revolución aprobados por el VI Congreso del Partido Comunista de Cuba.

Palabras clave: cooperativa, género, lineamientos y marco jurídico.

Abstract: The aim of this study is to provide some comments about the current situation of the relationship the gender and the cooperative within the frameworks within which the cooperative develops in Cuba, as the one that at the moment has been promulgated with experimental character, like part of the restructuring of the economic model provided in the Guidelines of the Economic and Social Policy of the Party and the Revolution adopted by the sixth Congress of the Communist Party of Cuba.

Key words: cooperative, gender, guidelines and legal framework.

* Máster en Derecho Constitucional y Administrativo, Profesora de Teoría General del Derecho y Derecho Romano, Departamento de Derecho, Facultad de Ciencias Sociales y Humanísticas, Universidad de Pinar del Río, Cuba. E-mail: oriselha@fcsh.upr.edu.cu 


\section{Una introducción necesaria}

El planteamiento de género en el sector productivo y laboral ha tenido grandes progresos pero aun no alcanza los niveles que permitan afirmar la perfecta consecución de una igualdad que parta del respecto a las diferencias naturales que existen entre mujeres y hombres y sus consecuentes roles.

El ámbito de lo productivo ha estado, y aún está, regido por reglas dictadas en función de la eficiencia y la ganancia, lo que supone un tratamiento incompleto de los principios de respeto y promoción de la mujer, ya que más bien promueven su asimilación a modos de actuar e ideales de éxito masculinos.

La regulación legal de las cuestiones de género no ha podido transformar las disposiciones respecto a la igualdad en un cambio real de las conductas dentro del ámbito laboral en un mundo dominado por el «eficientismo productivo», a pesar de que casi todas las normativas actuales han reconocido, en términos generales, la aspiración de alcanzar un tratamiento equitativo de género.

A pesar de estas limitaciones al Derecho le corresponde desempeñar un rol coadyuvante en la transformación de esta situación, rebasando las simples concepciones normativistas y poniéndose al servicio de la promoción, desde el plano legal, de alternativas que posibiliten un cambio en la conciencia social de la que forma parte ${ }^{1}$ en pos de desarrollar «su posible función transformadora». ${ }^{2}$

Ante esta situación se presenta una forma de organización, producción y apropiación que promueve, o puede promover, desde su diseño, importantes valores agregados a la eficiencia productiva y la socialización de la producción, entre lo que puede estar un mejor tratamiento de género: las cooperativas.

En Cuba la realidad género-cooperativa presenta características propias y crecientes posibilidades a la luz de la ampliación de los sectores en que se pueden implementar las cooperativas dentro de la econo-

1 «...el Derecho forma parte de la conciencia social y dentro de ella ocupa un lugar singular: las ideas sobre el Derecho, la expresión del fenómeno jurídico en la conciencia social se identifica como la conciencia jurídica, que no es más que la apreciación de la sociedad sobre los principales valores jurídicos, es decir, sobre la justicia, lo justo, lo legal, lo ilegal, etc.» Fernández Bulté, J., Teoría del Estado y el Derecho. Segunda Parte Teoría del Derecho, Editorial Félix Varela, La Habana, 2004, p. 22.

2 Masino, M.A., "Los aportes de Antonio Gramsci para una epistemología materialista del Derecho», publicado en Introducción a la epistemología del Derecho. Dirección de Publicaciones, Universidad Nacional del Rosario, 1988, p. 124. 
mía nacional. Por ello resulta oportuno valorar las posibles implicaciones que estas transformaciones pueden traer a esta relación.

\section{Potencialidades de las cooperativas para desarrollar un adecuado enfoque de género}

Para una comprensión más cabal del término género debe aclararse su connotación y su diferencia respecto a la categoría sexo. Se entiende por sexo al conjunto de características físicas, biológicas y corporales que definen, dentro del género humano, a hombres y mujeres. En tanto que género designa al conjunto de características psicológicas, sociales y culturales socialmente asignadas a las personas. Estas características son históricas, se van formando con el tiempo y por tanto son modificables lo que implica que «la perspectiva de género no suprime las diferencia entre hombres y mujeres». ${ }^{3}$

La complejidad las cuestiones asociadas a la perspectiva de género explican la diversidad de aproximaciones y criterios de tratamiento que han existido al respecto. En pos de alcanzar un enfoque integrado se ha recurrido el mainstreaming, que constituye la más reciente y completa opción para el tratamiento de género, una vez que se ha arribado al convencimiento de que solo es posible superar estos vicios históricos con un trabajo sistémico. Por mainstreaming se entiende «el proceso de valorar las implicaciones que tiene para los hombres y para las mujeres cualquier acción que se planifique, ya se trate de legislación, políticas o programas, en todas las áreas y en todos los niveles. Es una estrategia para conseguir que las preocupaciones y experiencias de las mujeres, al igual que las de los hombres, sean parte integrante en la elaboración, puesta en marcha, control y evaluación de las políticas y de los programas en todas las esferas políticas, económicas y sociales, de manera que las mujeres y los hombres puedan beneficiarse de ellos igualmente y no se perpetúe la desigualdad. El objetivo final de la integración es conseguir la igualdad de los géneros». ${ }^{4}$

La cooperativa, por su propia naturaleza, se presenta como una alternativa, altamente prometedora, al enfoque de género tradicional

3 Tshibilondi Ngoyi, A., La filosofía y los problemas de género en África. Temas

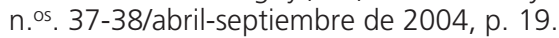

4 Glosario de términos relacionados con la transversalidad de género. Proyecto Equal «En clave de culturas», Secretaría Técnica del Proyecto Equal «En Clave de Culturas», 2007. 
pues tiene una proyección y alcances que claramente rebasan el ámbito de las relaciones laborales.

En su configuración, las cooperativas, se atienen a una serie de principios esenciales para garantizar que esta mantenga su esencia. Entre dichos principios figuran el de asociación abierta y voluntaria ${ }^{5}$, el de democracia ${ }^{6}$ y el de educación, capacitación e información ${ }^{7}$ que permiten completar un círculo de tratamiento integral de las cuestiones de género, si estas se enfocan adecamente, desde la inclusión de las socias, su participación y superación dentro del marco cooperativo, pues es impensable una entidad de asociados democráticamente organizados en la que no se considere medular la integración de todos sus miembros, con todas sus características, incluidas las de género, como elemento esencial para su credibilidad, legitimidad, eficiencia y eficacia.

Dentro de la lógica de funcionamiento que describen estos principios para la entidad cooperativa se pueden concebir formas alternativas a las imperantes de dar tratamiento a la cuestión de género, para lo cual es necesario que se haga de estos una adecuada regulación legal que permita su adecuada comprensión y realización.

Algunos criterios que se pueden tener en cuenta para el tratamiento de género dentro de las cooperativas se relacionan a continuación, sin que por ellos puedan considerarse como un catálogo cerrado. Las cooperativas pueden implementarlo, desde su naturaleza flexible, democrática y social, creando un beneficio, no solo empresarial con su desenvolvimiento, al hacer más activos y capaces a sus miembros, sino reportando, además, una importante contribución a la sociedad, al

5 «Las cooperativas son organizaciones voluntarias, abiertas para todas aquellas personas dispuestas a utilizar sus servicios y a aceptar las responsabilidades que conlleva la asociación, sin discriminación de género, raza, clase social, posición política o religiosa». ACl, «Declaración Sobre la Identidad Cooperativa», Manchester, septiembre de 1995, reproducida y comentada por El Hogar Obrero: Cooperativa de Consumo, Edificación y Crédito Ltda., en http://www.elhogarobrero1905.org.ar, consultada en diciembre de 2009.

6 «Las cooperativas son organizaciones democráticas controladas por sus asociados, quienes participan activamente en la definición de las políticas y en la toma de decisiones. Los hombres y mujeres elegidos para representar a su cooperativa responden ante los demás asociados. En las cooperativas de base las personas tienen igual derecho de voto (un asociado, un voto), mientras las organizaciones cooperativas de otros niveles también están organizadas con base en procedimientos democráticos». $\mathrm{ACl}$, ob. cit.

7 «Las cooperativas brindan educación y capacitación a sus asociados, a sus dirigentes electos, gerentes y empleados, de tal forma que contribuyan eficazmente al desarrollo de sus cooperativas. Las cooperativas informan al público en general, particularmente a jóvenes y creadores de opinión, acerca de la naturaleza y beneficios del Cooperativismo.» $\mathrm{ACl}, \mathrm{ob}$. cit. 
propiciar con su incidencia en el enfoque de género un tratamiento integral.

- En la cooperativa, como los estatutos no se imponen desde fuera sino que son de creación colectiva, se pueden adecuar a las necesidades y exigencias, particulares y cambiantes, del colectivo que la integra, incluidas las mujeres.

- La realización del principio de igualdad permite a las mujeres asumir responsabilidades y ejercitarse en la participación por medio de la emisión de criterios y proposición de ideas, valorizando sus aportes, con lo que se les prepara para devenir en ciudadanas activas, se les dignifica y da reconocimiento.

- Tiene la posibilidad de desarrollar actividades de promoción, educación e inclusión pues la naturaleza del objeto social de estas, dada su amplitud, les permite realizar diferentes acciones en pos de la superación de las mujeres miembros, así como llevar a cabo campañas de divulgación para la comunidad.

- Puede instituir horarios flexibles para la realización del trabajo, que sin afectar la eficiencia de la productividad, permitan el desarrollo de todas las actividades necesarias para el pleno desenvolvimiento de sus miembros mujeres ${ }^{8}$.

- Debe prever para la celebración de sus reuniones de un grupo de condiciones que permitan hacer efectiva y eficiente la participación de las mujeres, entre estas deben considerarse la cercanía y accesibilidad del lugar y horarios que respeten sus otras responsabilidades.

8 «La conciliación —entendida como algo igualitario tanto para mujeres como para hombres - es otro de los aspectos claves en los que las cooperativas dan el ejemplo...»:

«(...) es importante la flexibilidad a la hora de poner reuniones», concluye GóMEZ. "Nosotros teníamos muy claro cuando formamos la empresa que debíamos dejar tiempo para todo. Ahora, por ejemplo, gestiono la agenda de mis reuniones para que coincida cuando voy a Sevilla. No es algo que me impongan a cualquier hora y cualquier día, como sucedería con un jefe. Son valores añadidos que no nos los da una empresa capitalista».

«Resulta de interés reseñar que mientras en 2004 las mujeres directivas, en su mayor número, no tenían hijos, actualmente estas mujeres tienen dos hijos», concluía COCETA en la presentación del último estudio sobre la mujer, en 2009. "Cabría, tal vez, pensar que ello es debido a un progreso en la conciliación de la vida familiar y laboral, que posibilita que las mujeres puedan acceder a categorías laborales más cualificadas, asumiendo la maternidad de una forma responsable».

Vilnitzky, M. et al.: Mujer y cooperativismo, consultado en http://www.empresay trabajo.coop/024/dos02401.asp en fecha 27 de abril de 2012. 
De lo expuesto se colige que:

- Las cooperativas tienen una especial aptitud para enfocar en su desarrollo un tratamiento de las cuestiones de género desde una perspectiva más favorable que otras formas empresariales, pues desde la configuración misma de sus principios están favorablemente condicionadas, solo es cuestión de aprovecharla de forma coherente y consecuente, partiendo para ello de una regulación legal afín con sus principios.

Estas potencialidades no son indiferentes a la realidad cubana, que ha armonizado la inclusión de la figura cooperativa con una importante labor en el tratamiento de las cuestiones de género en los últimos 50 años.

\section{Situación actual de la relación género y cooperativa en Cuba}

La inclusión de la cooperativa en Cuba, con posterioridad al triunfo de la Revolución, es un resultado del carácter socialista adoptado por el Estado cubano y cuyo fin va dirigido a superar «...la división de los hombres en poseedores de los medios de producción y desposeídos de ellos» ${ }^{9}$. A tal propósito debe contribuir esta forma de propiedad a la par que fomenta «las relaciones personales [que] se basen en la colaboración y ayuda mutua» ${ }^{10}$.

La cooperativa en Cuba es reconocida por el artículo $20^{11}$ del magno texto cubano como un tipo de propiedad que «...constituye una forma avanzada y eficiente de producción socialista», a pesar de lo

9 Álvarez Tabío, F., Comentarios a la Constitución socialista cubana, Ed. Pueblo y Educación, La Habana, 1989, p. 84.

10 Ibidem.

11 «Los agricultores pequeños tienen derecho a asociarse entre sí, en la forma y con los requisitos que establece la ley, tanto a los fines de la producción agropecuaria como a los de obtención de créditos y servicios estatales.

Se autoriza la organización de cooperativas de producción agropecuaria en los casos y en la forma que la ley establece. Esta propiedad cooperativa es reconocida por el Estado y constituye una forma avanzada y eficiente de producción socialista.

Las cooperativas de producción agropecuaria administran, poseen usan y disponen de los bienes de su propiedad, de acuerdo con lo establecido en la ley y en sus reglamentos.

Las tierras de las cooperativas no pueden ser embargadas ni gravadas y su propiedad puede ser transferida a otras cooperativas o al Estado, por las causas y según el procedimiento establecido en la ley.

El Estado brinda todo el apoyo posible a esta forma de producción agropecuaria.» 
cual se ha limitado en su implementación solo al especio rural'12. Es por ello que para comprender la relación particular cooperativa-género en este terreno, especialmente delimitado, se hace necesario examinar la situación de las féminas en el campo, sector que dentro de la sociedad, en su conjunto, presenta características y rasgos propios especialmente en cuanto a roles asignados.

La vida en las zonas rurales de la Isla ha experimentado grandes mejorías, muchas de las cuales tienen una incidencia directa en las labores tradicionalmente femeninas: "Se han creado servicios básicos de apoyo al hogar, así como se ha continuado la distribución de efectos electrodomésticos, que facilitan las labores hogareñas y con ello también incentivan compartir las tareas del hogar en mejores condiciones. Se han concluido alrededor de 300 obras sociales, 45 policlínicos reparados y ampliados, amplio presupuesto se ha derivado para obras de alto impacto social. Más de 3 mil acueductos que garantizan el agua potable clorada han sido». ${ }^{13}$

Las mujeres eran el $47 \%$ de la población rural en $2010^{14}$. En cifras su presencia en el sector cooperativo campesino puede resumirse al porcentaje que representan del total de los socios de las diversas formas cooperativas existentes, siendo así que «en las CPA era el 17,4\% en 1999; en las CCS el 8,2\% en igual año; el 18,5\% en las UBPC agropecuarias y el $16,5 \%$ en las cañeras» ${ }^{15}$ de forma que "actualmente son socias 39623 mujeres, con iguales oportunidades, derechos y posibilidades que los socios hombres» ${ }^{16}$. Estas cifras no son desdeñables, pero

Constitución de la República de Cuba, consultada en http://www.parlamento cubano.cu/index.php?option=com_content\&view=article\&id=1418\&ltemid=83 en fecha 25 de mayo de 2012.

12 Sobre el debate al respecto al carácter agrario exclusivo de la cooperativo en Cuba «...las interpretaciones doctrinales al texto constitucional cubano de 1976 habían estado divididas (...) Para algunos «la Constitución reconoce a la cooperativa como una propiedad agraria, sin hacerla extensiva a ningún otro tipo de explotación económica», viéndose así —en la definición constitucional de la cooperativa - un límite para la expansión legal de la figura a otros sectores de la economía; para otros, en cambio, «la enumeración constitucional de las formas de propiedad no es taxativa, por lo cual es factible que la ley establezca la autorización para otras modalidades o posibilidades de la propiedad cooperativa.» Rodríguez Musa, O., ob. cit, p. 66.

13 Informe de Cuba a la Mesa Directiva sobre la mujer de América Latina y el Caribe de la CEPAL. Informe de Cuba, Santiago, Chile, 2010, publicado en www.eclac.cl/mujer/ noticias/noticias/1/41081/Cuba.pdf y consultado en fecha 20 de mayo de 2012.

14 Anuario Estadístico de Cuba 2010, publicado en http://www.one.cu/aec2010/ esp/03_tabla_cuadro.htm y consultado el 30 de mayo de 2012.

15 Pérez Rolo, Marta y Díaz, Elena, ob. cit., p. 67.

16 Informe de Cuba..., ob. cit. 
si se comparan con los porcentajes de féminas insertadas en otros sectores de la vida del país contrastan inmediatamente.

En pos de coadyuvar a la incorporación de la mujer a las labores del sector agropecuario la Asociación Nacional de Agricultores Pequeños (ANAP) «creó la Cátedra de Género en la Escuela Nacional de Capacitación, a través de la que se transversaliza la enseñanza de dicho enfoque para todos los planes y programas, en los diferentes cursos que allí se imparten para los cuadros y dirigentes de base de esa Asociación» ${ }^{17} .18$

En el plano legal ordinario las normas ordenadoras de las cooperativas cubanas - Ley n. ${ }^{\circ} 36$ Cooperativas Agropecuarias de 22 de julio de 1982 que fuera derogada por la Ley n. 95 Cooperativas de Producción Agropecuaria y de Créditos y Servicios de 2 de noviembre de 2002 y el Decreto Ley 142 Sobre las Unidades Básicas de Producción Cooperativa de 20 de septiembre de 1993- no han ofrecido una regulación única de los principios cooperativos, aunque han articulado una lógica de funcionamiento en torno a principios como el de democracia cooperativista, solidaridad humana y unión de los intereses personales con los colectivos en la cooperativa ${ }^{19}$ que ciertamente podría perfeccionarse tomando como referencia aspecto tratados en la Declaración sobre la Identidad Cooperativa de 1995.

En las citadas legislaciones se ha reconocido que pueden formar parte de ellas, sin distinción de sexo, «el cónyuge, hijos y demás familiares del cooperativista» ${ }^{20}$ de los afiliados y que estas se proyectaran en su trabajo hacia el «mejoramiento de las condiciones de vida de los cooperativistas y sus familiares... [promoviendo] la participación consciente de sus miembros en las tareas económicas y sociales de la nación, la lo-

17 Ibidem.

18 Otras organizaciones cuya labor se materializa en el sector agrario también han instrumentado estrategias de género. V. gr.: «La Asociación Cubana de Producción Animal (ACPA), presidida por una mujer, cuenta asimismo con una Estrategia de Género, la cual se aplica en todas las estructuras. Tiene instituido el Premio de la Mujer Rural, el que significa un estímulo para destacar a aquellas que se desempeñan en el campo científico, técnico y práctico de producción agroalimentaria. La ACTAF, Asociación Cubana de Técnicos Agrícolas Forestales tiene también su Estrategia de Género, privilegiando el acceso de las mujeres a dicha actividad.» Informe de Cuba..., ob. cit.

19 Cfr. Rojas Jiménez, A.: «La cooperativa como institución agraria en Cuba: principios normativos que la sostienen», Boletín de la Asociación Internacional de Derecho Cooperativo, núm. 44/2010, Bilbao, pp. 67-82.

20 Artículo 15. Ley n. ${ }^{\circ} 36$ Cooperativas Agropecuarias, publicada en http://www. parlamentocubano.cu/index.php?option=com_content\&view=article\&id=252:ley-no-36cooperativas-agropecuarias\&catid=46:leyes\&ltemid=79 en fecha 28 de mayo de 2012 . 
calidad y la comunidad.» ${ }^{21}$ A la par que «...trabajan en la educación de sus miembros para el cumplimiento de los principios que las rigen, así como en su capacitación técnica y la formación de sus cuadros».22

Aun cuando toda la labor antes señalada y los resultados alcanzados son significativos, una investigación desarrollada en el este del país sobre la incorporación femenina al trabajo en áreas agrícolas, señaló como causas desestimulantes «la sobrecarga doméstica, la persistencia de conductas y actitudes discriminatorias hacia la mujer en el empleo, la poca capacitación para el empleo y la existencia de inadecuadas condiciones para el trabajo» ${ }^{23}$.

En el Estudio sobre los valores de género en dos cooperativas cubanas cuyos resultados publicaran Marta Pérez Rolo y Elena Díaz ${ }^{24}$ se hace evidente un contraste entre los logros alcanzados por las mujeres y la permanencia de concepciones cultural e históricamente construidas, que limitan su plena realización.

Pues si de un lado se observan logros en cuanto a que:

— «Se aprecia que hay una satisfacción creciente entre las mujeres por su participación social.» ${ }^{25}$

— «Se muestra un sentimiento de independencia» ${ }^{26}, \mathrm{y}$

- «Se observa una tendencia, más acentuada en las trabajadoras de la UBPC, a que las mujeres se autovaloren como capaces de desempeñar labores de dirección y técnicas. ${ }^{27}$

De otro se mantienen concepciones que hacen que:

— «En tiempos de zafra, ambos miembros de la pareja aumentan sus jornadas laborales, aunque permanece la sobrecarga femenina doméstica y familiar. ${ }^{28}$

- «La mayoría de las aspiraciones de las mujeres se dirigen a la esfera familiar y doméstica. ${ }^{29}$

21 Artículo 6. Ley n. ${ }^{\circ} 95$ Cooperativas de Producción Agropecuaria y de Créditos y Servicios, publicada en http://www.parlamentocubano.cu/index.php?option=com content\&view=article\&id=307:ley-no95-cooperativas-de-producion-agropecuaria-yde-creditos-y-servicios\&catid=46:leyes\&ltemid=79, y consultada en 29 de mayo de 2012.

22 Artículo 7, ibidem

23 Pérez Rolo, Marta y Díaz, Elena, ob. cit., pp. 67-68.

24 Cfr. Pérez Rolo, Marta y Díaz, Elena, ob. cit., pp. 80-90.

25 Pérez Rolo, Marta y Díaz, Elena, ob. cit., p. 86.

26 Idem.

27 Ibidem, p. 88.

28 Ibidem, p. 84.

29 Ibidem, p. 85. 
— «Se mantiene la imagen del hombre proveedor.» ${ }^{30}$

- «Se mantiene, incluso, en ellas, la ubicación de los hombres fuera de tareas típicamente femeninas de acuerdo a la división tradicional, incluyendo la atención a niños. $\rangle^{31}$

En palabras de las propias autoras del Estudio «a pesar de las dificultades que aún persisten en la incorporación femenina al empleo socialmente retribuido en el área rural, los obstáculos son más fuertes en la persistencia de tradiciones que en las posibilidades de participación a partir de la existencia real de una plataforma estructural que ofrece condiciones y oportunidades de transformación de roles. ${ }^{32}$

Esto está dado fundamentalmente porque "Sin desconocer la realidad de esos progresos concientes o impuestos por la sociedad, todavía una mayoría significativa de hombres del campo se comportan con una masculinidad hegemónica bien acentuada y asentada en los viejos patrones familiares aprendidos desde la infancia, en tanto las mujeres, también en una sorprendente mayoría, aún las que han superado esquemas tradicionales de vida, siguen sometidas al poder masculino, quizás no en igual grado y condición que sus antecesoras». ${ }^{33}$

Lo antes expuesto demuestra que las políticas para enfrentar las concepciones de género, y las consecuencias derivadas de ellas, en el campo cubano tienen un impacto más demorado que en otros sectores de la vida del país donde el camino andado permitiría un avance más acelerado del desarrollo de las potencialidades de género presentes en las cooperativas, tomando en cuenta los niveles de inserción, preparación y los cambios en cuanto a percepciones culturales sobre roles ya operados en ellos.

De forma que en cuanto a la relación género-cooperativa en Cuba puede afirmarse que:

- La cooperativa ha demostrado en el marco legal, cultural, social y político cubano que es posible experimentar un sensible cam-

30 Ibidem, p. 86.

31 Ibidem, p. 88.

32 Pérez Rolo, Marta y Díaz, Elena, ob. cit., p. 68.

33 Álvarez, M., Masculinidades cubanas: El machismo guajiro. Ponencia en la Primera Jornada Cubana de Estudio sobre masculinidades (versión digital CD) CENESEX, Ciudad de La Habana, 2006. Citado por Castañeda Pérez, I., Aproximación al estudio de la equidad de género en la ANAP: premisas para un diagnóstico, Tesis en opción al Título de Máster en Gestión y Desarrollo de Organizaciones Cooperativas, 2007, publicada en http://www.flacsoandes.org/dspace/bitstream/10469/1169/1/Aproximaci\%C3\%B3n\%20 al\%20estudio \%20de\%20la\%20equidad\%20de\%20g\%C3\%A9nero...\%20lsabel\%20 Casta\%C3\%B1eda.pdf y consultada en fecha 25 de mayo de 2012. 
bio en determinados enfoques de género, no obstante el hecho de que se haya desarrollado exclusivamente en el sector rural no ha permitido que esta despliegue con mayor amplitud sus posibilidades.

Razón por la que conviene examinar las posibilidades que tendría la relación género-cooperativa ante una normativa, como la que se ha adoptado con carácter experimental por medio del Decreto-ley 305 «De las cooperativas no agropecuarias», que permite la extensión de esta figura a otros sectores de la economía a tono con los Lineamientos de la Política Económica y Social del Partido y la Revolución aprobados en el VI Congreso del Partido Comunista de Cuba.

\section{Marco jurídico experimental de la cooperativa en Cuba. Posible incidencia en la relación género y cooperativa}

En los Lineamientos de la Política Económica y Social del Partido y la Revolución ${ }^{34}$, aprobados el 18 de abril de 2012 por el VI Congreso del Partido Comunista de $\mathrm{Cuba}^{35}$ como directrices para el perfeccionamiento del modelo socioeconómico cubano figuran bajo el título de "LAS COOPERATIVAS» cinco puntos, del 25 al 29, que incluyen a estas entidades como parte de un nuevo «MODELO DE GESTIÓN ECONÓMICA» con el que el país se propone elevar la productividad del trabajo.

Sobre estas bases se ha venido trabajado por parte de la Comisión Permanente de Implementación y Desarrollo de los Lineamientos cuyo Presidente, Marino Murillo, anunció el 31 de marzo de 2012 en reunión ampliada del Consejo de Ministros la creación experimental de cooperativas fuera del sector agropecuario, sosteniendo que para cada experimento se han diseñado los principios fundamentales, reservando el papel regulador del Estado y el Gobierno según corresponde ${ }^{36}$. Lo cual está en consonancia con 36 de los 313 Lineamientos aprobados, poniendo de relieve la importancia general que se le atribuye a la figura cooperativa.

34 En lo adelante Lineamientos.

35 Vid. Lineamientos de la Política Económica y Social del Partido y la Revolución, aprobados el 18 de abril de 2012 en http://www.prensa-latina.cu/Dossiers/Lineamientos VICongresoPCC.pdf

36 Cfr. Cuba: Aprueba consejo de Ministros creación experimental de cooperativas no agropecuarias. Tomado del Noticiero del Mediodía de la Televisión Cubana, publicado en http://www.youtube.com/watch?v=7FVzkQhfkTQ y consultado en fecha 20 de junio de 2012. 
Hasta hace muy poco las regulaciones con que el ordenamiento jurídico cubano cuenta en materia de cooperativas son, básicamente: con carácter general el artículo 20 de la Constitución de la República de 1976 (actualizada); con carácter supletorio los artículos del 145 al 149 del Código Civil, Ley 59 de 16 de julio de 1987 (en adelante, CC) y con carácter especial la Ley de Cooperativas de Producción Agropecuaria y de Créditos y Servicios, Ley 95 del 2 de noviembre de 2002 (en lo adelante LCPACS) y el Decreto Ley 142 de 20 de septiembre de 1993, Sobre las Unidades Básicas de Producción Cooperativa.

Recientemente, en fecha 11 de diciembre de 2012, la Gaceta Oficial de la República de Cuba en una edición extraordinaria publicó un conjunto de normas que en lo adelante constituirán, con carácter experimental, el marco jurídico para las cooperativas no agropecuarias. Se trata de dos Decretos-Leyes, un Decreto y dos Resoluciones. El Decreto-Ley n. ${ }^{\circ} 305$ «De las cooperativas no agropecuarias» se ocupa de sentar "las normas que regulan la constitución, funcionamiento y extinción de cooperativas en sectores no agropecuarios de la economía nacional» ${ }^{37}$. El Decreto-Ley n. 306 «Del régimen especial de seguridad social de los socios de las cooperativas no agropecuarias» como su nombre indica «establece un régimen especial de seguridad social dirigido a la protección de los cooperativistas asociados en cooperativas no agropecuarias» ${ }^{38}$. El Decreto n. 309 del Consejo de Ministros dispone el "Reglamento de las cooperativas no agropecuarias de primer grado». La Resolución n. ${ }^{\circ} 570$ del Ministerio de Economía y Planificación pone en vigor el procedimiento de licitación respecto a los bienes de un establecimiento estatal para su gestión por las cooperativas no agropecuarias y la Resolución n. ${ }^{\circ} 427$ del Ministerio de Finanzas y Precios ordena las particularidades relativas a la materia tributaria, precios, normas contables, y mecanismos de financiación.

El carácter experimental que presentan estas nomas apunta, en primer lugar, que se trata de una práctica sometida a estudio y posible perfeccionamiento, por ello, y en segundo orden, se hacen mucho más importantes los estudios y reflexiones que al respecto se realicen, a fin de que la legislación definitiva que se adopte supere todas las li-

37 Art. 1, Decreto-Ley n. 305 «De las cooperativas no agropecuarias», Gaceta Oficial n. 053 Extraordinaria de 11 de diciembre de 2012, MINJUS, La Habana, 2012, p. 249.

38 Art. 1, Decreto-ley n. ${ }^{\circ}$ 306: «Del régimen especial de seguridad social de los socios de las cooperativas no agropecuarias», Gaceta Oficial n. ${ }^{\circ} 053$ Extraordinaria de 11 de diciembre de 2012, MINJUS, La Habana, 2012, p. 254. 
mitaciones y fijen claras posiciones respecto a los múltiples aspectos del desarrollo cooperativo cubano que hoy se encuentran en debate.

Algunos de los principales aspectos relativos al desarrollo cooperativo que se encuentran sometidos a discusión en la actualidad, y que inciden directamente en el perfeccionamiento de dicha institución, son su naturaleza jurídica, los sectores de la economía en los cuales se desarrolla, los fines a cuyos efectos se concibe legalmente y su forma de funcionamiento. Estos son elementos que afectan directamente las potencialidades de la cooperativa para desarrollar un adecuado tratamiento en materia de género por lo que, también desde esta perspectiva, resultan relevantes.

\section{Naturaleza jurídica}

La naturaleza jurídica de la cooperativa ha sido enfocada desde variadas posiciones dentro de las que se pueden apuntar tres posiciones básicas: la que la considera una asociación; la que la considera una sociedad y la que la considera una categoría autónoma. ${ }^{39}$

Dentro del vigente ordenamiento jurídico cubano la cooperativa se ha concebido como una forma de propiedad, como lo demuestra el artículo 20 de la Constitución cubana al sancionar que «Esta propiedad cooperativa es reconocida por el Estado...», y el Código Civil al ubicarla en sU TíTULO II: DERECHO DE PROPIEDAD, CAPÍTULO ॥: FORMAS DE PROPIEDAD, bajo la denominación de la SECCIÓN TERCERA: Propiedad cooperativa.

La razón por la que la actual definición de la naturaleza jurídica de la cooperativa cobra relevancia para la materia de género, radica precisamente en la inviabilidad que le crea su reconocimiento como una forma de propiedad para poder garantizar un tratamiento de tales cuestiones, toda vez que limitan la figura a las relaciones de los propietarios respecto a un conjunto de bienes, y las que entre ellos se produzca con razón de las anteriores relaciones, conjurando cualquier otro tipo de finalidad o tipología de relación.

Una nueva realidad se nos ofrece con el Decreto-Ley 305/2012 que en sU ARTíCULO 2.1 reconoce a la cooperativa como «una organización con fines económicos y sociales, que se constituye voluntaria-

39 Vid. Gadea, E.; Sacristán, F. y Vargas Vasserot, C.: Régimen jurídico de la sociedad cooperativa del siglo XXI. Realidad actual y propuestas de reforma, Dykiston SL, Madrid, 2009, pp. 70-83. 
mente sobre la base del aporte de bienes y derechos y se sustenta en el trabajo de sus socios» ${ }^{40}$.

Esta definición de la naturaleza jurídica de la cooperativa resulta aún imprecisa pues su catalogación como «organización» no aporta ninguna claridad obvia, aunque del resto de las normas, en particular las del Capítulo II «De la constitución» ${ }^{41}$, se puede colegir que se le equipara a una sociedad mercantil.

A pesar de estas insuficiencias lo cierto es que abre paso a un mejor enfoque de la figura y, por ende, a que desde la persona jurídica resultante, en cualquier caso, se pueda concebir un espacio a la finalidad de tratar las cuestiones de género, algo que resulta difícil de concebir por parte de una forma de propiedad.

Por lo que se puede afirmar que:

- Sin una modificación de la legislación vigente, en cuanto a la determinación de la naturaleza jurídica de la cooperativa, la figura carece de posibilidad directa de tratar las cuestiones de género. De ahí que la recién promulga regulación experimental de la materia suponga un primer paso para el diseño coherente de la relación género-cooperativa, al permitir un asidero legal válido para su tratamiento.

Sector de la economía en que se desarrolla

En Cuba, como referíamos supra, el marco legal actual solo contempla la creación de cooperativas en el sector agropecuario de la economía.

A fin de rebasar esta sectorialización los Lineamientos eliminaron los adjetivos que denotan limitación en cuanto a la naturaleza de las actividades a desarrollar por las cooperativas, al establecer en su numeral 25 que «se crearán las cooperativas de primer grado como una forma socialista de propiedad colectiva, en diferentes sectores... ${ }^{42}$.

40 Art. 2.1, Decreto-Ley n. ${ }^{\circ}$ 305: «De las cooperativas no agropecuarias», Gaceta Oficial n. ${ }^{\circ} 053$ Extraordinaria de 11 de diciembre de 2012, MINJUS, La Habana, 2012, p. 249.

${ }^{41}$ El procedimiento de autorización que dispone y el requisito de inscripción en el Registro Mercantil, guarda grandes similitudes con otros procedimientos vigentes para la autorización de la actividad de empresarios mercantiles en Cuba.

42 Lineamientos de la Política Económica y Social del Partido y la Revolución, aprobados el 18 de abril de 2012 en http://www.prensa-latina.cu/Dossiers/Lineamientos VICongresoPCC.pdf 
Consecuente con tal política el Decreto-Ley 305 de 2012, y el resto de las normas que complementan sus regulaciones, abren paso a la implementación de cooperativas no agropecuarias.

En los análisis que motivó el debate de los Lineamientos quedó sentado que estas cooperativas deberían desarrollarse fundamentalmente en sectores que hoy ocupaban los trabajadores por cuenta propia ${ }^{43}$ y otros que hasta "ahora tutela el estado».44

Esta impresión parece corroborarse al examinar el ARTíCULO 6 del Decreto-Ley $n .^{\circ} 305$ de $2012^{45}$ que reconoce cuatro formas esenciales de surgimiento, las que evidencian que los campos fundamentales en los que se dará la creación de las nuevas cooperativas serán aquellos en los que se desempeñan las personas naturales y los que cubren entidades estatales en sectores que no se consideran vitales para el país, si se interpreta de forma orgánica con las finalidades del resto del ordenamientos jurídico.

En el Reglamento que las ordena se dispone, en su ARTíCULO 14, que «el objeto social de la Cooperativa comprende las producciones, prestación de servicios o la actividad de comercialización, a que se dedi-

43 «Al analizar el nuevo contexto, debemos entender el importante potencial que significan estos trabajadores individuales, pequeños y dispersos, para la configuración de las emergentes cooperativas cubanas. Ellos pudieran constituir en la ciudad, el sujeto homólogo del pequeño agricultor en el campo, salvando las diferencias relativas a la propiedad de los bienes que decidan aportarse a la actividad cooperada. Las razones económicas, políticas y sociales que aconsejan y justifican la instrumentación legal del derecho de asociarse en cooperativa a los segundos, debe valer — con igual fuerzapara los primeros, ya que —en última instancia — se trata de asegurar la eficacia del carácter socialista del texto constitucional cubano, ponderando la realización colectiva del derecho al trabajo...» Rodríguez Musa, O., ob. cit, pp. 77-78.

44 El presidente de la Comisión Constitucional de la Asamblea Nacional, José Luis Toledo, explica los Lineamientos, publicado en http://www.parlamentocubano.cu/ index. php?option=com_content\&view=article\&id=3336:el-presidente-de-la-comisionconstitucional-de-la-asamblea-nacional-jose-luis-toledo-explica-los-lineamientos y consultado en fecha 20 de junio de 2012.

45 «Las cooperativas de primer grado pueden formarse: a) A partir del patrimonio integrado por los aportes dinerarios de personas naturales que deciden voluntariamente asociarse entre sí bajo el régimen de propiedad colectiva. b) Por personas naturales que decidan voluntariamente asociarse entre sí, solo con la finalidad de adquirir conjuntamente insumos y servicios, comercializar productos y servicios, o emprender otras actividades económicas, conservando los socios la propiedad sobre sus bienes. c) A partir de medios de producción del patrimonio estatal, tales como inmuebles y otros, que se decida gestionar de forma cooperativa y para ello puedan cederse estos, por medio del arrendamiento, usufructo u otras formas legales que no impliquen la transmisión de la propiedad. d) Una combinación de las formas anteriores», Decreto-ley n. ${ }^{\circ} 305 \ldots$ ob. cit., p. 250. 
cará de acuerdo con lo que se autorice» ${ }^{46}$. Con ello quedan diseñadas, dentro del panorama cooperativo cubano, cooperativas de colocación de la producción ${ }^{47}$, cooperativas de trabajo ${ }^{48}$, y, aunque no resulta tan evidente en la primera lectura, cooperativas de distribución ${ }^{49}$, ya que el inciso b) del artículo 6 del Decreto-Ley n. 305 las concibe con «la finalidad de adquirir conjuntamente insumos y servicios» lo que sienta una interesante posibilidad ya que la DISPOSICIÓN ESPECIAL PRIMERA, del citado Reglamento, esclarece que no se limita solo a los cuentapropistas al efectuar una puntualización para aquellos «socios que sean trabajadores por cuenta propia» ${ }^{50}$.

A esta considerable ampliación de espacios y formas para el desarrollo cooperativo debe sumarse la instrumentación de las cooperativas de segundo grado que los Lineamientos proponen destinar a "actividades complementarias afines o que agreguen valor a los productos y servicios de sus socios (de producción, servicios y comercialización), o realizar compras y ventas conjuntas con vistas a lograr mayor eficiencia» ${ }^{51}$. Según la DISPOSICIÓN FINAL SEXTA del Decreto-Ley n. ${ }^{\circ} 305$ «El Consejo de Ministros, una vez que entre en vigor el presente Decreto-Ley, emite el Reglamento para las cooperativas de primer grado, y en el plazo de trescientos sesenta (360) días dictará el Reglamento para las cooperativas de segundo grado» ${ }^{52}$ lo que permite supone que a la vuelta de un año se estarán dando los primeros pasos para su creación.

El impacto de estas modificaciones en la relación género-cooperativa resulta evidente, pues afecta sectores donde la presencia y los roles desempeñados por la mujer tiene características sustancialmente diferentes al agropecuario. Aparejado a ello convierte a esta en una relación de peso creciente dentro de la realidad nacional, en proporción

46 Decreto n. 309 del Consejo de Ministros que dispone el «Reglamento de las cooperativas no agropecuarias de primer grado». Gaceta Oficial n. ${ }^{\circ} 053$ Extraordinaria de 11 de diciembre de 2012, MINJUS, La Habana, 2012, p. 263.

47 Agrupan productores y procuran colocar los resultados de sus producciones en las mejores condiciones posibles de precio, regularidad y seguridad. Vid. Kaplan de Drimer, A. y Drimer, B.: Las cooperativas. Fundamentos-Historia-Doctrina, Ed. Intercoop, Buenos Aires, 1981, pp. 156 y ss.

48 Reúnen a obreros, técnicos, profesionales, etc. que organizan en común su trabajo, a fin de proporcionarles fuentes de ocupación estables y convenientes. Vid. ibidem.

49 Proporcionan a sus asociados los artículos y servicios que estos necesitan, en las mejores condiciones posibles de calidad y precio. Vid. idem.

50 Decreto n. ${ }^{\circ} 309 \ldots$ ob. cit., p. 270.

51 Lineamiento 29, Lineamientos..., ob. cit.

52 Decreto-ley n. ${ }^{\circ} 305 \ldots$ ob. cit., p. 253. 
directa al aumento de las cooperativas, pues según crezca su número más importante se vuelve la forma en qué estas enfoquen las cuestiones de género debido a la cantidad de mujeres implicadas.

En primer lugar, si partimos de que una de las fuentes de las nuevas cooperativas será el empleo por cuenta propia, tendríamos una fuerza femenina en activo que era del $20 \%$ total de trabajadores en $2010^{53}$, cifra que ha aumentado con la ampliación de actividades experimentada por este sector de la economía tomando en consideración, V. gr., que entre los empleos más solicitados por los cuentapropistas «está la elaboración y venta de alimentos y la contratación de la fuerza de trabajo que le sirve de apoyo... ${ }^{54}$, o sea, la esfera de los servicios donde en 2010 las mujeres eran el 40,20\% de la fuerza de trabajo. ${ }^{55}$

Considerando que «El gobierno cubano prevé este año un incremento de 240.000 trabajadores no estatales, los que sumados a los actuales superarán el medio millón.... ${ }^{56} \mathrm{y}$, aplicando un cálculo proporcional en base a cifras similares a las antes mencionadas, podemos entender que la cantidad de féminas incorporadas sería, desde el inicio, superior al que actualmente se vinculan a las formas cooperativas tradicionales.

En el sector estatal, del que se puedan desgajar algunas entidades para el sector cooperativo, se ha partido de dar prioridad a los antiguos trabajadores ${ }^{57}$ con lo que la presencia femenina tendrá también un peso significativo. Una presencia además marcada por un considerable nivel de calificación toda vez que las mujeres son el 43,3\% de la fuerza laboral y el $66,1 \%$ de la fuerza técnica. ${ }^{58}$

Por ello puede considerarse que:

- La situación de los niveles de calificación alcanzados por las mujeres en Cuba, unido a su nivel de presencia en el sector del cuentapropismo, permiten comprender el alto nivel de participa-

53 Anuario Estadístico de Cuba 2010, publicado en http://www.one.cu/aec2010/ esp/07_tabla_cuadro.htm y consultado en fecha 20 de junio de 2012.

54 Número de cuentapropistas sigue creciendo en Cuba, publicado en http:// www.cubadebate.cu/noticias/2012/04/01/numero-de-cuentapropistas-sigue-creciendoen-cuba/ y consultado en fecha 20 de junio de 2012.

55 Anuario Estadístico..., ob. cit.

56 Número de cuentapropistas..., ob. cit.

57 Art. 10.2: "Cuando la forma de la cooperativa sea la prevista en el inciso c) del artículo 6 del presente Decreto-Ley, tendrán preferencia para ser socios fundadores los trabajadores de esas entidades.» Decreto-Ley n. ${ }^{\circ} 305 \ldots$, ob. cit., p. 250.

58 Caram León, T., Empoderamiento femenino en Cuba. Criterios para su análisis en las Cooperativas. FLACSO-Cuba, p. 1, publicado en http://www.flacso.uh.cu/sitio_ revista/num2/articulos/art_TCaram.pdf, consultado en fecha 25 de mayo de 2012. 
ción que estas pueden alcanzar en las nuevas cooperativas que se conformen a partir de las normas experimentales.

- Se abre una nueva era a la relación género-cooperativa con mayor importancia social y un contexto sustancialmente diferente al anterior, que puede ser el punto de partida para que todas las potencialidades descritas de flexibilidad, inclusión, empoderamiento y valorización presentes en las cooperativas se llevaran a un nivel superior dentro de la realidad cubana.

Fines a cuyos efectos se concibe legalmente

Directamente conectado a lo anterior aparece la cuestión de la finalidad que legalmente se le reconozca a las cooperativas, de formas que estas cuenten con aptitud para desarrollar las actividades que, fuera de lo estrictamente productivo, se les pueden demandar.

Aunque el texto de los Lineamientos hizo una mención en extremo limitada y economicista de los fines cooperativos al adjudicarles únicamente «...la finalidad de producir y prestar servicios útiles a la sociedad.... ${ }^{59}$, las normas experimentales han ampliado el espectro al contemplar que si bien su «objetivo general es la producción de bienes y la prestación de servicios mediante la gestión colectiva» con esto procura «la satisfacción del interés social y el de los socios»60. Como complemento, el artículo 4 inciso f) formula un principio denominado Responsabilidad social, contribución al desarrollo planificado de la economía y al bienestar de sus socios y familiares, el cual, en su párrafo 2 do, sostiene que las cooperativas «Trabajan por fomentar la cultura cooperativista y por las satisfacciones de las necesidades materiales, de capacitación, sociales, culturales, morales y espirituales de sus socios y familiares». ${ }^{61}$

Estas normas crean el marco oportuno para la inserción, implícita como contribución social y para la satisfacción de los socios y sus familias, de la finalidad de género por medio de otros fines mayores que de conjunto implican la realización de sus potencialidades en tal sentido.

Las nuevas perspectivas que presenta la cooperativa en los sectores no agropecuarios se desarrollan en una realidad marcada por grandes progresos institucionales, políticas favorables al pleno desarrollo femenino, que contrastan con la cultura media marcada por la subsis-

\footnotetext{
59 Numeral 25 de los Lineamientos.

60 Artículo 2.1, Decreto-ley n. ${ }^{\circ} 305 \ldots$, ob. cit., p. 249.

61 Artículo 4.f), ibidem, p. 250.
} 
tencia de prácticas y concepciones de roles tradicionales. La labor de la Federación de Mujeres Cubanas (FMC) ha sido vital en la promoción de las políticas más exitosas. Muchas cifras pueden dar testimonio de los resultados alcanzados, en el plano de la salud (mortalidad materna 29,9 RMM $\left.{ }^{62}\right)$, la esperanza de vida al nacer (77,97 años con una sobrevida femenina de 4,02 años $\left.{ }^{63}\right)$, la formación académica y la ocupación profesional. No obstante, esto no implica que se haya logrado superar la clásica división de funciones entre roles pues se mantiene "sobre ella (...) la carga doméstica y familiar a pesar de su protagonismo social». ${ }^{64}$ Situación que se agudizó con la crisis económica a que se enfrentó el país desde principios de los 90 y que aún continua, aunque ha experimentado una distensión en los últimos años con el impacto directo en la vida doméstica de políticas estatales dirigidas a mejorar las condiciones de las labores hogareñas.

Precisamente por tales razones:

- Corresponde a la cooperativa, en el marco del reconocimiento de las nuevas finalidades que le confieren la legislación experimental, sentar pautas en el tratamiento diferenciado e integral de la cuestión de género dentro del espacio que ella constituye y sobre la base de los principios que la inspiran, a fin de que la relación género-cooperativa aporte una nueva perspectiva para el desarrollo integral de las féminas.

\section{Funcionamiento}

Todas estas posibilidades finalmente se deben concretar en el funcionamiento de las cooperativas, donde deben materializarse por medio de un tratamiento integral de género.

El funcionamiento de las cooperativas puede entenderse en dos planos, el interno, que comprende las relaciones que se dan entre los

62 Este es índice del periodo 2000-2004. (RMM = Razón de Muerte Materna por 100 000 nacidos vivos.) Fuente: 1950-1959: Anuario Demográfico; 1960-1979: Formulario de la OPS; 1980-2004: Dirección Nacional de Estadísticas, MINSAP. Citada por Cabezas Cruz, Evelio, "Evolución de la mortalidad materna en Cuba». Revista Cubana Salud Pública, Ciudad de La Habana, v. 32, n. 1, marzo de 2006. Disponible en http://scielo.sld. cu/scielo.php?script=sci_arttext\&pid=S0864-34662006000100005\&lng=es\&nrm=iso, Consultada el 19 de mayo de 2012.

63 MINSAP, Anuario Estadistico de Salud 2010. Consultado en http://files.sld.cu/dne/ files/2011/04/anuario-2010-e-sin-graficos1.pdf, en fecha 26 de mayo de 2012.

64 Ibidem. 
socios, y el externo que abarca lo tocante a su relación como persona jurídica con otras personas naturales o jurídicas. En ambas dimensiones debe proyectar igualmente una perspectiva de género.

A lo interno esto se refiere al trato de los socios y socias, en el cual se perfilan los regímenes de participación, tanto en las aportaciones de capital como de trabajo, la toma de decisiones, la distribución de las ganancias y en la organización del conjunto de actividades que se desarrollen. Hacia lo externo se materializa en su impacto en la comunidad y el resto de la sociedad en general, y como regla obedece a la labor educativa y de capacitación, que hace las veces de enlace entre uno y otro plano.

A fin de instrumentar las alternativas que se convengan para dar tratamiento a cuestiones de género las cooperativas requieren, como punto de partida de su funcionamiento, de autonomía e independencia que les permita gestionar por sí mismas las estrategias que determinen.

En tal sentido la nueva legislación experimental cubana dispone un marco para el funcionamiento signado, hasta donde lo conciben las normas publicadas, por la autonomía y la determinación colectiva de los socios ${ }^{65}$, por lo que solo resta contrastar las posibilidades con la práctica a partir de que se creen las primeras experiencias.

Cabe formular importante expectativas si se parte de que la situación de roles en este sector estatal y del trabajo por cuenta propia es diferente al que se da en el campo, toda vez que «...muchas de ellas, [actúan como] las verdaderas dueñas y gerentes de sus establecimientos, aunque sus esposos aparecían formalmente como tales». ${ }^{66}$ De hecho, "... hay una gran diferencia entre el sector informal cubano y sus rasgos en otros países; por la calificación de estas mujeres, el papel protagónico que desarrollan y porque el trabajo informal no se ejerce para lograr un nivel de supervivencia...». ${ }^{67}$

Las particularidades de los sectores y fuentes de que han de nutrirse las nuevas cooperativas a desarrollarse en Cuba permiten esperar que:

- En el funcionamiento de las cooperativas se perciban distintos niveles de empoderamiento femenino y que se implementen nuevas formas de articular los roles, dando un tratamiento coherente a las cuestiones de género e incluso la aparición de formas integradas netamente por féminas.

65 Cfr. Decreto n. ${ }^{\circ} 309 \ldots$ ob. cit.

66 Pérez Rolo, Marta y Díaz, Elena, ob. cit., p. 66.

67 Ibidem, pp. 67-68. 


\section{A modo de conclusiones}

Dentro del mundo laboral se hace necesario que las formas de organización y planificación del mismo, partiendo de la legislación particular de la actividad que se trate, se adapten aún más a las necesidades de pleno desarrollo de las mujeres, de forma que la realización personal y profesional de ellas no supongan una disyuntiva excluyente.

Las cooperativas suponen un importante espacio de desarrollo de potencialidades en cuanto al enfoque de género toda vez que están pensadas para el mejor desarrollo de sus miembros y de la sociedad en general, sin que la parte económico-productiva de las mismas pueda desnaturalizar su esencia humanista y socialista.

En la actualidad Cuba presenta una importante labor en materia de género, incluido el sector cooperativo, pero la limitación de esta solo a las labores agropecuarias, donde las concepciones tradicionales de los roles se muestran más resistentes, no ha permitido que se exploten al máximo sus potencialidades a pesar de las políticas llevadas a cabo con este fin.

La legislación experimental adoptada en materia de cooperativa, de conformidad con los Lineamientos de la Política Económica y Social del Partido y la Revolución, representa, a pesar de las imperfecciones que pueda tener, un significativo avance a fin de ampliar la presencia de las cooperativas en nuestra economía, articulando principios y finalidades que sientan las bases para una implementación práctica que supere limitaciones anteriores. Por tal razón cabe esperar que los resultados en materia de género sean cualitativamente superiores toda vez que en los sectores en que desarrollarían el nivel de formación y de capacidad demostrada de la féminas garantizaría un nivel superior de partida, y la preparación y actitud de estas es ideal para hacer un uso más acabado de las posibilidades de desarrollo personal-profesional que las cooperativas les ofrecen.

\section{Bibliografía}

\section{Textos}

Astelarra, J.: «Estado y Políticas de género», publicado en Temas n.os 37-38/ abril-septiembre de 2004.

Álvarez Tabío, F.: Comentarios a la Constitución socialista cubana, Ed. Pueblo y Educación, La Habana, 1989.

AzCuY, H.: "Análisis de la Constitución cubana», en Revista Papeles de la FIM n. ${ }^{\circ}$ 14, Madrid, 2000. 
CabezAs Cruz, Evelio: "Evolución de la mortalidad materna en Cuba». Revista Cubana Salud Pública, Ciudad de La Habana, v. 32, n. 1, marzo de 2006. Disponible en <http://scielo.sld.cu/scielo.php?script=sci_arttext\&pid=S0864$34662006000100005 \&$ lng=es\&nrm=iso $>$. Consultada el 19 de mayo de 2012.

CARAM LeÓn, T.: Empoderamiento femenino en Cuba. Criterios para su análisis en las Cooperativas. FLACSO-Cuba, p. 1, publicado en http://www.flacso. uh.cu/sitio_revista/num2/articulos/art_TCaram.pdf, consultado en fecha 25 de mayo de 2012.

CASTAÑeda Pérez, I.: Aproximación al estudio de la equidad de género en la ANAP: premisas para un diagnóstico, tesis en opción al Título de Máster en Gestión y Desarrollo de Organizaciones Cooperativas, 2007, publicada en http://www.flacsoandes.org/dspace/bitstream/10469/1169/1/ Aproximaci\%C3\%B3n\%20al\%20estudio\%20de\%20la\%20equidad\%20 de\%20g\%C3\%A9nero...\%20lsabel\%20Casta\%C3\%B1eda.pdf y consultada en fecha 25 de mayo de 2012.

Fernández Bulté, J.: Teoría del Estado y el Derecho. Segunda Parte Teoría del Derecho, Editorial Félix Varela, La Habana, 2004.

GAdEA, E.; SACRISTÁn, F. y VARgas VASSEROt, C.: Régimen jurídico de la sociedad cooperativa del siglo XXI. Realidad actual y propuestas de reforma, Dykiston SL, Madrid, 2009.

Kaplan De Drimer, A. y Drimer, B.: Las cooperativas. Fundamentos-Historia-Doctrina, Ed. Intercoop, Buenos Aires, 1981.

MAsino, M.A.: «Los aportes de Antonio Gramsci para una epistemología materialista del Derecho», publicado en Introducción a la epistemología del Derecho. Dirección de Publicaciones, Universidad Nacional del Rosario, 1988.

MINSAP: Anuario Estadístico de Salud 2010. Consultado en http://files.sld.cu/ dne/files/2011/04/anuario-2010-e-sin-graficos1.pdf, en fecha 26 de mayo de 2012.

Orellana Zambrano, Waldo E.; Celis Ekstrand, Amanda: «Género y capacidades directivas en cooperativas de trabajo asociado. Estudio exploratorio en ctas valencianas», en REVESCO. Revista de Estudios Cooperativos, n. ${ }^{\circ} 105$, Universidad Complutense de Madrid, 2011, pp. 13-33.

TSHIBILONDI NGoYI, A.: "La filosofía y los problemas de género en África», en Temas n. ${ }^{\text {os }}$ 37-38/abril-septiembre de 2004.

Pérez Rolo, Marta y DíAz, Elena: "Estudio sobre los valores de dirección y de género en las cooperativas cubanas», en Cuaderno pedagógico, volumen 2IRECUS FLACSO/CUBA, Université de Sherbrooke Universidad de La Habana, uniRcoop, 2006.

PiñeIRo HaRneCKer, C. (compiladora): Cooperativas y socialismo: una mirada desde Cuba, Centro Martin Luther King, La Habana, 2011.

Rodríguez Musa, O.: La cooperativa como figura jurídica. Perspectivas constitucionales en Cuba para su aprovechamiento en otros sectores de la economía nacional diferentes al agropecuario. Dikinson S.L., Madrid, 2011.

RodríGuez ReYes, I.: «Relaciones de género en la familia cubana: ¿espacio de igualdad o desigualdades?», en Temas n. ${ }^{\circ}$ 48, octubre-diciembre de 2006. 
SÁnCheZ VÁZqueZ, A.: "Reflexiones — iintempestivas? — sobre la igualdad y la desigualdad», en Entre la realidad y la utopía. Ed. de Ciencias Sociales, La Habana.

VILNITZKY, M. et al.: Mujer y cooperativismo, consultado en http://www.empresa ytrabajo.coop/024/dos02401.asp en fecha 27 de abril de 2012.

\section{Otros documentos}

ACI: «Declaración sobre la identidad cooperativa», Manchester, septiembre de 1995, reproducida y comentada por El Hogar Obrero: Cooperativa de Consumo, Edificación y Crédito Ltda.», en http://www.elhogarobrero1905.org.ar, consultada en diciembre de 2009.

Anuario Estadístico de Cuba 2010, publicado en http://www.one.cu/aec2010/ esp/03_tabla_cuadro.htm y consultado el 30 de mayo de 2012.

Cuba: Aprueba consejo de Ministros creación experimental de cooperativas no agropecuarias. Tomado del Noticiero del Mediodía de la Televisión Cubana, publicado en http://www.youtube.com/watch?v=7FVzkQhfkTQ y consultado en fecha 20 de junio de 2012.

El presidente de la Comisión Constitucional de la Asamblea Nacional, José Luis Toledo, explica los Lineamientos, publicado en http://www.parlamento cubano.cu/index. php?option=com_content\&view=article\&id=3336:elpresidente-de-la-comision-constitucional-de-la-asamblea-nacional-jose-luistoledo-explica-los-lineamientos y consultado en fecha 20 de junio de 2012.

Glosario de términos relacionados con la transversalidad de género. Proyecto Equal «En clave de culturas», Secretaría Técnica del Proyecto Equal «En Clave de Culturas», 2007.

Informe de Cuba a la Mesa Directiva sobre la mujer de América Latina y el Caribe de la CEPAL. Informe de Cuba, Santiago, Chile, 2010, publicado en www.eclac.cl/mujer/noticias/noticias/1/41081/Cuba.pdf y consultado en fecha 20 de mayo de 2012.

Lineamientos de la Política Económica y Social del Partido y la Revolución, aprobados el 18 de abril de 2012 en http://www.prensa-latina.cu/Dossiers/ LineamientosVICongresoPCC.pdf

Número de cuentapropistas sigue creciendo en Cuba, publicado en http:// www.cubadebate.cu/noticias/2012/04/01/numero-de-cuentapropistassigue-creciendo-en-cuba/ y consultado en fecha 20 de junio de 2012.

\section{Legislación}

Constitución de la República de Cuba, consultada en http://www.parlamento cubano.cu/index.php?option=com_content\&view=article\&id=1418\&ltemid $=83$ en fecha 25 de mayo de 2012.

Ley n. ${ }^{\circ} 36$ Cooperativas Agropecuarias, publicada en http://www.parlamento cubano.cu/index.php?option=com_content\&view=article\&id=252:ley- 
no-36-cooperativas-agropecuarias\&catid=46:leyes\&ltemid=79 en fecha 28 de mayo de 2012.

Ley n. ${ }^{\circ} 49$ Código de Trabajo, publicada en http://www.parlamentocubano.cu/ index. .php?option=com_content\&view=article\&id=264:ley-no-49-codigode-trabajo\&catid=46:leyes\&ltemid=79 y consultada en fecha 30 de mayo de 2012.

Ley n. 95 Cooperativas de Producción Agropecuaria y de Créditos y Servicios, publicada en http://www.parlamentocubano.cu/index.php?option=com_ content\&view=article\&id=307: ley-no95-cooperativas-de-producionagropecuaria-y-de-creditos-y-servicios\&catid=46:leyes\&ltemid=79, y consultada en fecha 29 de mayo de 2012.

Decreto Ley n. ${ }^{\circ} 234$ de 2003 De la maternidad de las trabajadoras, publicado en http://www.sld.cu/galerias/pdf/sitios/insat/dl-234-2003.pdf y consultada en fecha 30 de mayo de 2012.

Decreto-Ley n. ${ }^{\circ} 305$ «De las cooperativas no agropecuarias», Gaceta Oficial n. 053 Extraordinaria de 11 de diciembre de 2012, MINJUS, La Habana, 2012.

Decreto-Ley n. ${ }^{\circ} 306$ «Del régimen especial de seguridad social de los socios de las cooperativas no agropecuarias», Gaceta Oficial n. ${ }^{\circ} 053$ Extraordinaria de 11 de diciembre de 2012, MINJUS, La Habana, 2012.

Decreto n. ${ }^{\circ} 309$ del Consejo de Ministros que dispone el «Reglamento de las cooperativas no agropecuarias de primer grado». Gaceta Oficial n. 053 Extraordinaria de 11 de diciembre de 2012, MINJUS, La Habana, 2012.

Resolución n. ${ }^{\circ} 570$ del Ministerio de Economía y Planificación, Gaceta Oficial n. 053 Extraordinaria de 11 de diciembre de 2012, MINJUS, La Habana, 2012.

Resolución n. ${ }^{\circ} 427$ del Ministerio de Finanzas y Precios, Gaceta Oficial n. ${ }^{\circ} 053$ Extraordinaria de 11 de diciembre de 2012, MINJUS, La Habana, 2012. 